DOI: $10.4274 /$ tpa.322

\title{
Tuberosclerosis cases presenting with cardiac mass during the neonatal period
}

\author{
Demet Terek, Özge Altun Köroğlu, Mehmet Yalaz, Mert Kazandı, Şule Gökçe, Murat Deveci, \\ Ertürk Levent, Mete Akisu, Ruhi Özyürek, Nilgün Kültürsay \\ Ege University Medical Faculty, Department of Pediatrics, Izmir, Turkey
}

\begin{abstract}
Summary
The aim of the study is to evaluate the tuberosclerosis cases diagnosed during the neonatal period presenting with cardiac masses. The clinical and laboratory findings of 4 cases and of tuberosclerosis diagnosed in Ege University Medical Faculty Newborn Clinic were evaluated retrospectively. The first case was admitted to the Newborn Clinic with the diagnosis of cardiac mass detected at the $28^{\text {th }}$ gestational week. Cranial magnetic resonance imaging revealed subependimal hamartomas. Echocardiography showed large masses within the intraventricular cavity and cardiac apex. The second case was admitted to the Newborn Clinic with the diagnosis of cardiac mass detected at the $29^{\text {th }}$ gestational week. Cranial magnetic resonance imaging revealed subependimal hamartomas and cortical tubers. Echocardiography showed multiple masses located at right ventricular outflow, right atrium and left ventricule. In the third case multiple cardiac masses in both ventricules were detected during the neonatal period. Cranial magnetic resonance imaging showed multiple tubers. The fourth case was admitted to the Newborn Clinic with the diagnosis of cardiac mass detected at the $32^{\text {th }}$ gestational week. Three hipopigmented skin lesions were found. Echocardiography showed multiple masses within the right ventricle, left ventricle and interatrial septum. Cranial magnetic resonance imaging revealed subependimal nodules. Cardiac mass should suggest Tubeous Sclerosis, cranial imaging must be performed. (Turk Arch Ped 2013; 48: 57-61)
\end{abstract}

Key words: Cardiac mass, intrauterine, tuberoslerosis

\section{Introduction}

Primary cardiac tumors are observed rarely in the childhood age group. The most common cardiac tumor in the childhood age group and intrauterine period is rhabdomyoma (RM). RMs in the heart may lead to cardiac failure and hydrops fetalis in the prenatal period and stillbirth. In the neonatal period, it may cause cardiac murmur, cardiac failure and arrhyhthmia or may be asymptomatic. However, surgical treatment may be performed if it leads to mechanical obstruction or life-threatening arrhythmias. It has been reported that the most common cardiac finding of tuberosclerosis is rhabdomyomas with a rate of $50-80 \%$. TS should be considered in patients in whom RM is found in the heart because of their common association. In this study, information about intracardiac masses and TS is presented because of four newborn subjects who were found to have intracardiac masses on echocardiographies (ECO) performed three times in the intrauterine period and once in the neonatal period and diagnosed as tuberosclerosis with investigations performed in the postnatal period.

\section{Case}

Case 1. It was learned that a female patient who was born by cesarean section at the 40-41st gestational week from a 22-yearold G1P0 healthy mother with a birth weight of $3510 \mathrm{~g}$ was followed up because of cardiac mass found at the 28th gestational week (Figure 1). Fetal ECHO revealed an appearance of hypertrophy and mass in the interventricular septum and apex. There was no familial history of known disease. On physical examination, no pathology was found except for a 2/6 murmur heard especially in the pulmonic area. Complete blood count and biochemical tests were within normal limits. Electrocardiogram (ECG) revealed sinus ryhthm. Echocardiography revealed a large mass in the interventricular septum and apex and normal valve functions. The mass did not 
cause obstruction in the ventricular outlet tract. The patient was investigated in terms of TS considering rhabdomyoma. Cranial and abdominal ultrasonographies were found to be normal. The supratentorial section on cranial magnetic resonance (MR) revealed lesions related to subependimal and periventricular hamartomas in the head of caudate nucleus and around the foramen monro which were hyperdense in the T1-weighted images and hypodense in the T2-weighted images at the level of bilateral centrum semiovale and lateral ventricules with a higher number and larger dimension in the left (Figure 2) and it was reported that these findings supported TS. On opthalmologic examination, a hypopigmentated vegetative lesion was found in the optic disc of the right eye which was considered to be inside the vitreum. Thereupon, orbital computarized tomography (CT) was performed. A noncalcified lesion with a dimension of approximately $2 \times 3 \mathrm{~mm}$ was found in the bulbus oculi at the level where the optic nerve enters the bulbus. The appearance was found to be compatible with astrocytic hamartoma when evaluated with brain findings. Electroencephalogram (EEG) revealed no pathology. The patient was monitorized and followed up in terms of arrhythmia. Surgical intervention was not considered, since the mass did not disrupted the hemodynamic balance and the patient was discharged without any problem to come back for follow-up visits.

Case 2. It was learned that a female patient who was born at the 38th gestational week by cesarean section from a 31-yearold G2P1 healthy mother was followed up because of cardiac mass found on ECHO performed at the 29th gestational week. The first child of the family was found to have aortic coartation and hypoplastic left heart syndrome in the neonatal period and was lost on the fifth day of life because of sudden cardiac arrest. Physical examination was found to be normal. Complete blood count and biochemical tests were found to be normal. Cranial and abdominal ultrasonographies were found to be normal. Electrocardiogram revealed sinus ryhthm. On echocardiogram, a mass with dimensions of $8 \times 15 \mathrm{~mm}$ in the right ventricular outlet tract, a mass with dimensions of $8 \times 14 \mathrm{~mm}$ in the right atrium localized posteriorly which did not compress the inferior and superior vena cava and multiple masses in the left ventricular wall, in the mitral anterior papillary muscle and right ventricular apex with the largest one having a dimenson of $8 \mathrm{~mm}$ were found. Since the findings were compatible with multiple rhabdomyomas, the case was investigated in terms of TS. The supratentorial sections on cranial MRI revealed diffuse hamartomas in the subependimal white matter which were hyperdense on T1-weigted images and hypodense on T2weighted images, hamartomas at the level of the foramen monro and suspicious $\mathrm{T} 1$ hyperdense lesions which might be related to cortical tubers on T1-weighted images. With these findings the patient was diagnosed as TS. Ophtalmological examination was found to be normal. Paroxysmal disorder composed of spike waves was found in the occipital regions of the right hemisphere on EEG examination in the patient who had no seizures clinically. Antiepileptic treatment was not started. Surgical intervention was not considered in the patient, either, since the mass did not disrupt the hemodynamics and did not lead to arrhythmia. The patient who was monitorized and found to have no arrhythmia was discharged to come back for follow-up visits.

Case 3. A female patient who was born at the 37-38th gestational week from a G2P1 healthy mother was started to be followed up because of a cardiac mass found on fetal ECHO. There was no pathology in her personal and familial history. Physical examination revealed no pathology except for a 1/6 systolic murmur heard in the pulmonic area. Complete blood count and biochemical tests were found to be normal. Abdominal and cranial ultrasonographies were found to be normal. Electrocaridogram revealed sinus rhythm. On echocardiogram, a total of three hyperechogenic masses were found in the right and left ventricles near the apex with the one in the left ventricle having a dimension of $12 \times 8 \mathrm{~mm}$ and the larger one of the other two ones in the right ventricle having a dimension of $6 \mathrm{~mm}$. Cranial MRI was performed in the patient who was considered to have rhabdomyoma. Nodules typical for tuberosclerosis were found adjacent to both foramina of Monro. A tuber with a diameter of $5 \mathrm{~mm}$ was also found in the left frontal region. With these findings TS was considered and the patient

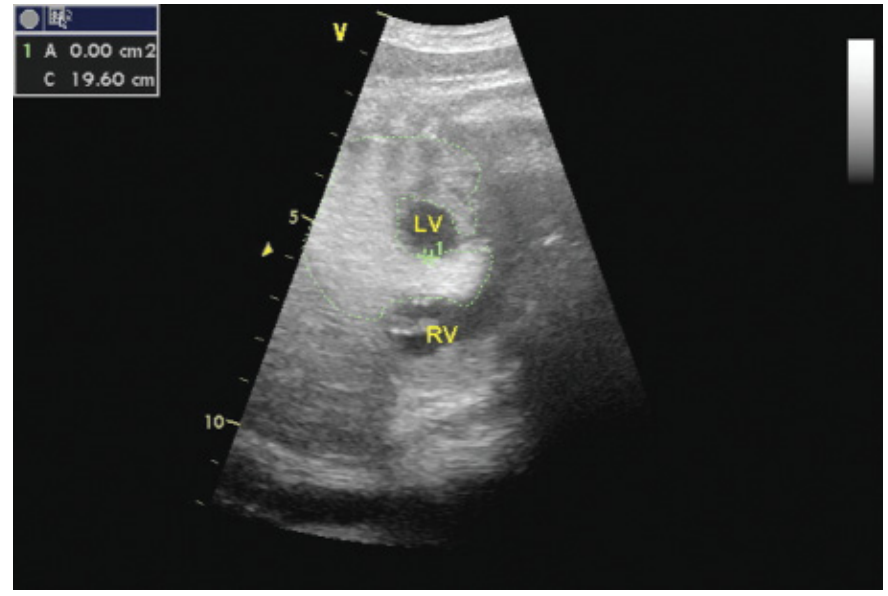

Figure 1. Appearance of the tumor on fetal echocardiogram

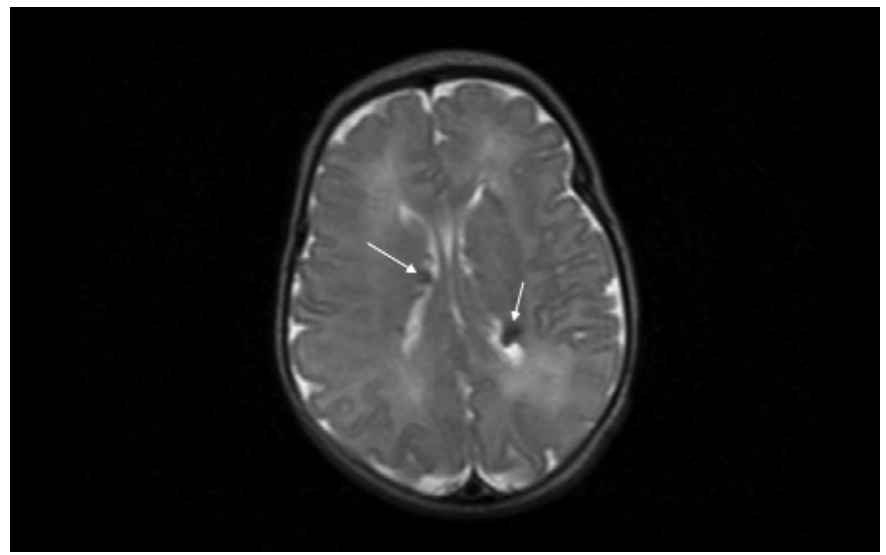

Figure 2. Appearance of hamartomas on magnetic resonance imaging 
was monitorized. Ophtalmological examination was found to be normal. Electroencephalogram was found to be normal. The patient who had no problem or arrhythmia in the follow-up was discharged to come back for follow-up visits. In the outpatient follow-up visits, it was observed that the caridac masses disappeared.

Case 4. A male patient who was born at term with a birth weight of $4040 \mathrm{~g}$, referred to our center because of myocardial thickening found on prenatal ultrasonography at the 32-33rd gestational week and found to have an appearance compatible with RM on fetal ECHO performed at the 34th gestational week was started to be followed up. There was no familial history of known disease. Physical examination revealed no pathology except for a 2/6 systolic murmur in the mesocardium and three hypopigmented lesions on the trunk. Complete blood count and biochemical tests were found to be normal. Electrocardiogram revealed sinus ryhthm. On postnatal ECHO, three-four masses with the largest one having a diameter of $1.2 \mathrm{~cm}$ were found in the right ventricle, five-six masses with the largest one having a diameter of $1 \mathrm{~cm}$ were found in the left ventricle and a mass having a diameter of $5 \mathrm{~mm}$ was found in the interatrial septum (Figure 3). On abdominal ultrasonography, cystic formations were found in both kidneys and the lesions in the kidneys were evaluated as millimetric simple cysts smaller than $1 \mathrm{~cm}$ on abdominal tomography. Cranial MRI revealed subependimal nodules in the bilateral ventricular walls and mass lesion in the parenchyma especially prominent in the left hemisphere. Paroxysmal activity originating from the frontotemporal part of the left hemisphere and temporocentral part of the right hemisphere was observed on EEG in the patient who had no seizures. The patient who had no problem or arrhythmia in the follow-up was discharged to come back for follow-up visits. Antiepileptic treatment was started in the patient who had a seizure at the age of five months and was followed up by the neurology department. Follow-up ECHOs revealed that the cardiac masses were reduced in size.

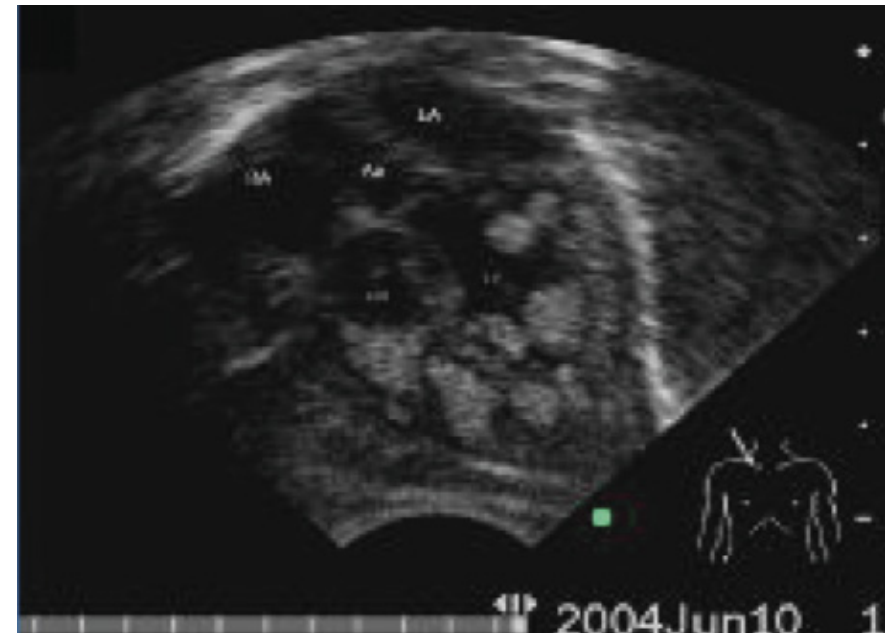

Figure 3. Appearance of the cardiac masses on ECHo performed at the first hour postnatally.

\section{Discussion}

In echocardiography studies, the frequency of primary cardiac tumors in the childhood age group was found to be $0.17 \%$ (1). A great majority of primary cardiac tumors are benign. Rhabdomyoma $(60 \%)$, teratoma $(25 \%)$ and fibroma $(12 \%)$ are the primary cardiac tumors which may be observed in the childhood. They develop in the intrauterine period and may be diagnosed early with prenatal ultrasonography. They may lead to fetal arrhythmia, non-immune hydrops fetalis, respiratory distress, cardiac failure and cyanosis or they may be asymptomatic. The rate of asymptomatic rhabdomyoma has been calculated to be 1/326 000 in infants (1). The relation of cardiac rhabdomyomas with TS is well known. It has been reported that cardiac RMs may be one of the earliest signs of TS $(2,3)$. Our patients were diagnosed in the early period by investigations performed because of cardiac mass found in the intrauterine period without a familial history. Cardiac rhabdomyomas are observed with a rate of $60 \%$ below the age of two in patients with a diagnosis of TS (4). The frequency of these tumors in the neonatal period is $1 / 40000$. Cardiac rhabdomyomas in the intrauterine period appear as homogeneous, round and hyperechogenic masses on USG. The ones associated with tuberosclerosis are mostly in the right ventricle and multiple. It was shown that cardiac rhabdomyomas might have dimensions ranging between $4 \mathrm{~mm}$ and $55 \mathrm{~mm}$ and the tumors enlarged markedly in the second and third trimesters in the intrauterine period $(8,10)$. Both atrial and ventricular masses were found in our second and fourth patients and only ventricular masses were found in our fist and third patients. While three of our patients had multiple caridac masses, our first patient had a large single mass as thickening in the interventricular septum and ventricular walls.

Cardiac rhabdomyomas may be asymptomatic or may be manifested by clinical and hemodynamical findings depending on the number of tumors, localization of the tumor and dimensions of the tumor. In the intrauterine period, they may be found on fetal ultrasonographic screenings or may be manifested as hydrops fetalis. In the postnatal period, they may be completely asymptomatic or they may be manifested by a murmur alone or congestive heart failure, decreased cardiac output due to intracardiac obstructions and arrhythmia and sudden death may occur (1). When the diameter of the tumor mass exceeds $20 \mathrm{~mm}$, the perinatal mortality risk of the fetus is high (10). Arrhythmias, supraventricular and ventricular tachycardia and atrioventricular blocks may generally be observed with rhabdomyomas $(6,11)$. It is thought that the tumor contains embrionic Purkinje cells histopathologically and these constitute an accessory conduction pathway between the atrium and ventricle and cause ventricular preexcitation and arrhythmias $(4,12)$. It has been shown that symptomatic rhabdomyomas have a mortality rate of $53 \%$ in the first week $(6,13)$. In our four patients, cardiac complaints and arrhythmia findings on ECG were absent. 
In cardiac rhabdomyomas, reduction generally occurs as the age gets older (4). In some studies, they have been shown to regress partially at the age of two and completely at the age of four. In one study, complete regression was found in $28 \%$ of the cardiac tumors, partial regression was found in $46 \%$ and no change was found in $19 \%(6,14)$. In our third patient, the cardiac masses were observed to be disappeared at the age of two.

Treatment is symptomatic. Since reduction occurs most of the time, medical or surgical treatment options are considered depending on the hemodynamics, cardiac pathology and the localization of the tumor. Antiarrhythmic agents may be used in treatment (8). Surgical treatment is recommended in case of mechanical obstruction in the heart or life-threatening arrhythmias. Surgical intervention was not performed in our patients, since they were asymptomatic and their hemodynamic states were not disrupted.

It has been reported that $\mathrm{ECHO}$ is important and adequate in the prenatal diagnosis and follow-up of cardiac rhabdomyomas $(4,10)$. Other cardiac tumors which should be considered in the differential diagnosis ultrasonographically include fibroma, mixoma, teratoma and hemangioma. Sonographically, it is not possible to differentiate rhabdomyoma, fibroma and mixoma. Teratomas appear as masses outside the heart and are associated with pericardial effusion. Hemangiomas contain cystic and solid areas and they occur in the right atrium. The diagnosis is made between the 21st and 30th gestational weeks by ultrasonography.

Although fetal ECHO in cases of familial TS is normal at the 18th gestational week, masses may be found in the fetal heart at the 22nd gestational week (15). Since the tumors in the kidneys and brain can not be observed by ultrasonography in these cases, fetal MRI may be performed and the family may be informed about termination of pregnancy within legal limits by explaining neurologic sequelas and the risk of fetal loss (15). An association between cardiac rhabdomyoma and trisomy 21,13 and 18 has been reported; karyotyping by amniocentesis may be performed after interviewing with the family. Molecular diagnosis can be made in pregnant women with a familial history of TS (16).

Tuberosclerosis is a disese which occurs as a result of spontaneous mutations which inactivate the TSC1 $(9 q 34,3)$ and TSC2 $(16 \mathrm{p} 13,3)$ genes coding two proteins called tuberin and hamartin (4). It has an autosomal dominant inheritance (5). Its prevalence has been reported to be approximately one in 6000 12000 live births. $60-80 \%$ of the cases occur with new mutations (6). In rare cases, TSC2 mutation is more frequent. TCS1 and TCS2 mutations have not been found in $20 \%$ of the patients with a marked phenotype for tuberosclerosis (7). The disease involves multiple systems. The brain and skin are affected most commonly. The kidney, eye, lung, bone and heart are also affected. Clinically, resistant epilepsy, mental retardation, behavioral problems and skin lesions are observed. Convulsions are the most common reason for presentation and start mostly in the first year of life. In our study, one patient had a seizure at the age of four months and one patient had a seizure at the age of five months and antiepileptic treatment was started. Genetic studies could not be performed in our patients becasue of economic problems.

The prominent brain lesion is calcified or non-calcified tuber localized subependimally in the brain hemisphere. Important findings in the diagnosis include subependimal giant-cell astrocytoma and subependimal nodules. Our first patient had hamartamatous lesions and our second, third and fourth patients had subependimal nodules and tubers.

Hypopigmented lesions which are cutaneous findings have an oval or leave-like shape and different dimensions. They are mostly observed in the trunk and extremities. They become prominent in the first years of life. They may not be observed in the neonatal period, since pigmentation is not developed and may appear in time. Only one of our patients had hipopigmented lesion on the body.

The most common renal lesions in tuberosclerosis include angiolipomas and renal cysts. However, renal carcinomas may also occur. The frequency of renal cysts is not known. One of our patients had bilateral renal cysts.

Patients with tuberosclerosis may have ophtalmological findings including retinal tumors, astrocytic hamartoma, pigment changes in the iris and colobomas. Our first patient had retinal astocytic hamartomas.

It should be kept in mind that cardiac rhabdomyoma in the neonatal period may be associated with TS even though other clinical findings of TS are absent and diagnostic laboratory tests should be performed.

\section{References}

1. Ibrahim $\mathrm{CPH}$, Thakker P, Miller PA, Baro D. Cardiac rhabdomyoma presenting as left ventricular outflow tract obstruction in a neonate. Interact CardioVasc Thorac Surg 2003; 2(4): 572-574.

2. Amonkar GP, Kandalkar BM, Balasubramanian M. Cardiac rhabdomyoma. Cardiovasc Pathol 2009; 18(5): 313-314.

3. Lacey SR, Donofrio MT. Fetal cardiac tumors: prenatal diagnosis and outcome. Pediatr Cardiol 2007; 28(1): 61-67.

4. Uzun H, Yavuz T, Arslanoğlu i. Supraventriküker taşikardi ile kendini gösteren üç aylık tüberoskleroz olgusu. Bakırköy Tıp Dergisi 2009; 5: 75-77.

5. İncecik F, Hergüner Ö, Özcan K, Altunbaşak A. Tuberosklerozlu 19 olgunun geriye dönük olarak değerlendirilmesi. Türk Ped Arş 2006; 41(3): 156-160.

6. Gürses D, Levent E, Ülger Z, Özyürek AR. Fetal ekokardiyografi ile kalpte rabdomiyomların saptandığı tuberosklerozlu bir olgu sunumu. Türk Ped Arş 2009; 44(4): 146-148.

7. Jozwiak J, Jozwiak S, Wlodarski P. Possible mechanism of disease development in tuberous sclerosis. Lancet Oncol 2008; 9: 73-79.

8. Misirlioğlu ED, Aliefendioğlu D, Alpan N, Güven A, Peltek N. Case 3: Fetal intracardiac tumour-A feature of a genetic disease? Acta Paediatr 2006; 95(6): 758-761.

9. Degueldre SC, Chockalingam P, Mivelaz Y, Di Bernardo S, Pfammatter JP, Barrea C, Sekarski N, Jeannet PY, Fouron JC, Vial Y, Meijboom EJ. Considerations for prenatal counselling of patients with cardiac rhabdomyomas based on their cardiac and neurologic outcomes. Cardiol Young $2010 ; 20(1)$ : 18-24.

10. Chao AS, Chao A, Wang TH. Outcome of antenatally diagnosed cardiac rhabdomyoma: case series and a meta-analysis. Ultrasound Obstet Gynecol 2008 ; 31(3): 289-295. 
11. Butany J, Nair V, Naseemuddin A, Nair GM, Catton C, Yau T. Cardiac tumors: diagnosis and management. Lancet Oncol 2005; 6(4): 218-228.

12. Gillette PC, Blair HL, Crawford FA. Preexcitation syndromes. In: Gillette PC, Garson A (ed)s. Pediatric cardiac dysrhythmias. New York: Grune and Stratton, 1981: 360-379.

13. Fenoglio JJ Jr, MCAllister HA Jr, Ferrans VJ. Cardiac rhabdomyoma: a clinicopathologic and electron microscopic study. Am J Cardiol 1976; 38(2): $241-251$.
14. Mühler EG, Turniski-Harder V, Engelhardt W, von Bernuth G. Cardiac involement in tuberous sclerosis. B Heart J 1994(6); 72: 584-590.

15. Allan L, Hornberger LK, Sharland G. Fetal cardiac tumors. Textbook of fetal Cardiology 2000; 358-365.

16. Jó wiak S, Doma ska-Pakie a D, Kwiatkowski DJ, Kotulska K. Multiple cardiac rhabdomyomas as a sole symptom of tuberous sclerosis complex: case report with molecular confirmation. J Child Neurol 2005; 20(12): 988-989. 